\title{
Spatiotemporal structures in the internally pumped optical parametric oscillator
}

\author{
Lodahl, Peter; Bache, Morten; Saffman, Mark
}

Published in:

Physical Review A

Link to article, DOI:

10.1103/PhysRevA.63.023815

Publication date:

2001

Document Version

Publisher's PDF, also known as Version of record

Link back to DTU Orbit

Citation (APA):

Lodahl, P., Bache, M., \& Saffman, M. (2001). Spatiotemporal structures in the internally pumped optical parametric oscillator. Physical Review A, 63(2), 023815.1-023815.12.

https://doi.org/10.1103/PhysRevA.63.023815

\section{General rights}

Copyright and moral rights for the publications made accessible in the public portal are retained by the authors and/or other copyright owners and it is a condition of accessing publications that users recognise and abide by the legal requirements associated with these rights.

- Users may download and print one copy of any publication from the public portal for the purpose of private study or research.

- You may not further distribute the material or use it for any profit-making activity or commercial gain

- You may freely distribute the URL identifying the publication in the public portal

If you believe that this document breaches copyright please contact us providing details, and we will remove access to the work immediately and investigate your claim. 


\title{
Spatiotemporal structures in the internally pumped optical parametric oscillator
}

\author{
P. Lodahl, ${ }^{1}$ M. Bache, ${ }^{1,2}$ and M. Saffman ${ }^{3}$ \\ ${ }^{1}$ Optics and Fluid Dynamics Department, Risb National Laboratory, Postbox 49, DK-4000 Roskilde, Denmark \\ ${ }^{2}$ Department of Mathematical Modelling, Technical University of Denmark, DK-2800 Lyngby, Denmark \\ ${ }^{3}$ Department of Physics, University of Wisconsin, 1150 University Avenue, Madison, Wisconsin 53706
}

(Received 31 August 2000; published 17 January 2001)

\begin{abstract}
We analyze pattern formation in doubly resonant second-harmonic generation in the presence of a competing parametric process, also named the internally pumped optical parametric oscillator. Different scenarios are established where either the up- or down-conversion processes dominate the spatiotemporal behavior. The possibility of obtaining exact solutions above threshold for the parametric oscillation process allows detailed analytical investigations of the parametric instability, that are supplemented by numerical analysis. We identify secondary instabilities that lead to formation of negative patterns and gray solitons. Estimates of the thresholds for pattern formation under experimentally relevant conditions are given.
\end{abstract}

DOI: 10.1103/PhysRevA.63.023815

PACS number(s): 42.65.Sf, 42.65.Ky, 42.65.Tg

\section{INTRODUCTION}

Pattern formation in cavity enhanced $\chi^{(2)}$ nonlinear processes has been a flourishing research area recently. Patterns are formed due to transverse instabilities in the plane perpendicular to the propagation direction. Two fundamentally different processes have been described that are the optical parametric oscillator (OPO) [1-3] and second-harmonic generation (SHG) [4-6]. The spontaneous formation of patterns has been attributed to an off-axis emission mechanism mediated by the nonlinearity and diffraction when the intracavity fields are detuned slightly from cavity resonance. The detuning can either be introduced manually by scanning the cavity length or, in the case of SHG, occur as a nonlinear effect. The absence of nonlinear phase shifts in the OPO appears since patterns are generated directly at the parametric oscillation threshold while an oscillation threshold does not exist in SHG. This is the major reason for the differences between SHG and OPO.

Pattern formation under the combined processes of SHG with competing nondegenerate parametric oscillations has been treated recently both for singly resonant $[7,8]$ and doubly resonant [9] SHG. This combined system, also named the internally pumped optical parametric oscillator (IPOPO), was shown to be of experimental relevance by Schiller et al. [10] in their work on efficient frequency doubling. Hence, the parametric fields can be generated in such a way that they automatically obey resonance conditions in the cavity, which lowers the oscillation threshold. This additional decay process substantially alters the classical [11] as well as the quantum $[12,13]$ behavior of SHG. The competing parametric process turns out to be of great relevance also for studies of pattern formation in SHG.

Here we describe the formation of spatiotemporal structures in doubly resonant SHG under the influence of the competing parametric process. The parametric process is found to influence pattern formation in two fundamentally different ways. For some parameters the instabilities seen in a pure SHG system, with the parametric process neglected, can exist, while completely different transverse structures are observed in other regions. The SHG instabilities were previ- ously studied by Etrich et al. [4] but the analysis presented here is generalized to include also the possibility of nonideal phase matching of the frequency conversion process in addition to unequal propagation distances for the fundamental and second harmonic. These extra degrees of freedom turn out to be usable tuning parameters in order to reach new parameter regions, especially for tuning the SHG instabilities with respect to the parametric threshold.

The instabilities due to the parametric process are studied in detail in this paper. The simplicity of the parametric instability allows derivation of exact solutions valid above the parametric threshold. Such exact solutions were previously found in the externally pumped nondegenerate OPO by Longhi [3] and by Marte in the IPOPO without diffraction [11]. Depending on the sign of the fundamental detuning, the parametric fields are either emitted as homogeneous off-axis or on-axis waves in the cavity. None of these instabilities lead to any spatially modulated intensity structure, but while the off-axis parametric solutions are proven to be linearly stable and hence quench pattern formation, the on-axis solutions can destabilize through a secondary instability. This instability was previously found to lead to formation of complicated spatiotemporal structures, as, e.g., intensity spiral patterns [14]. Here we will focus on other secondary instabilities of the IPOPO including a parametric bistability and a self-pulsing instability. They are found to lead to novel transverse structures such as honeycomb patterns and oscillating dark solitons.

In addition to these studies of the rich pattern formation dynamics in the IPOPO, we also analyze a realistic experimental setup in order to address the question of the experimental realizability of the scheme. This is particularly relevant in light of the recent progress in the experimental realization of pattern formation in $\chi^{(2)}$ based resonators [15].

\section{BASIC MODEL}

\section{A. The configuration}

Figure 1 shows the cavity configuration for doubly resonant SHG. The cavity consists of two independent arms for the fundamental and the second-harmonic fields, respec- 


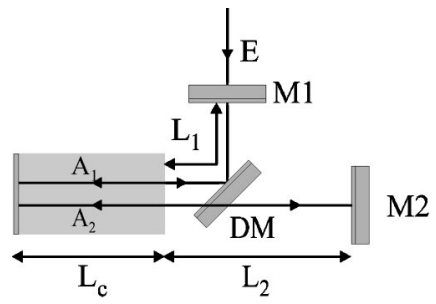

FIG. 1. Doubly resonant SHG cavity geometry.

tively. We discuss a semimonolithic standing-wave geometry where one end of the nonlinear $\chi^{(2)}$ crystal serves as mirrors for both fields, and externally adjustable output coupling mirrors are used. The two fields are separated by a dichroic mirror that reflects the fundamental and transmits the second-harmonic. The cavity is pumped at the fundamental frequency through the input coupling mirror (M1) with an intensity transmission coefficient $T$, while the mirror M2 in the second-harmonic path is assumed to be highly reflecting. The two-arm configuration is advantageous since it allows independent tuning of the two frequencies and thus, from an experimental point of view, double resonance can be more easily obtained as compared to monolithic devices. Independent tunable doubly resonant cavities have been described by $\mathrm{Ou}$ and Kimble [16]. In Fig. 1, $L_{c}$ is the crystal length and the propagation distances outside the nonlinear medium are denoted $L_{1}$ and $L_{2}$ for the fundamental and second harmonic, respectively. The parametric fields that can be excited in the system are not shown in the figure, but since phase-matching restrictions require them to be generated with frequencies close to the fundamental they will propagate in the same cavity arm.

A central point is to have a cavity with all transverse modes degenerate in order to obtain nonlinear boundary independent patterns in contrast to mode dynamics. We will investigate the plane-plane configuration but alternatively, e.g., a confocal geometry could have been chosen in order to enhance the nonlinear coupling with focused beams and eliminate the propagation losses occurring in the plane-plane cavity [7]. However, narrow focusing is in general not of interest since the spatial scale of the pattern under investigation might become too large compared to the width of the Gaussian beam and consequently the pattern can be suppressed. This point will be investigated further in Sec. V. In an experiment it could be favorable to use a stable cavity, and close to degeneracy it might still be possible to see features of pattern formation dynamics as pointed out by Valcárcel [17].

\section{B. Mean-field model}

Mean-field equations for the IPOPO can be derived from the paraxial wave equation. The equations hold under the assumptions of small cavity round-trip losses and detunings. The derivation will not be given here but is very similar to the one presented in Ref. [7] in the case of singly resonant SHG. The following description will concern the specific experimental setup illustrated in Fig. 1. Experimental work on this configuration is currently in progress. The analysis presented here includes effects due to potentially different round-trip propagation distances of the fundamental and second harmonic as well as different diffraction coefficients inside and outside the crystal. We obtain the following set of four coupled equations:

$$
\frac{\partial A_{1}}{\partial t}=\left(-1+i \Delta_{1}\right) A_{1}+f(\xi) A_{1}^{*} A_{2}+i \nabla_{\perp}^{2} A_{1}+E,
$$

$$
\begin{aligned}
\frac{\partial A_{2}}{\partial t}= & \left(-\gamma+i \Delta_{2}\right) A_{2}-\tau f^{*}(\xi) A_{1}^{2}-2 \tau f^{*}(\widetilde{\xi}) A_{+} A_{-} \\
& +\frac{i}{2} v \nabla_{\perp}^{2} A_{2}, \\
\frac{\partial A_{+}}{\partial t}= & \left(-1+i \Delta_{+}\right) A_{+}+f(\widetilde{\xi}) A_{-}^{*} A_{2}+i \nabla_{\perp}^{2} A_{+}, \\
\frac{\partial A_{-}}{\partial t}= & \left(-1+i \Delta_{-}\right) A_{-}+f(\widetilde{\xi}) A_{+}^{*} A_{2}+i \nabla_{\perp}^{2} A_{-},
\end{aligned}
$$

where $f(x)=i e^{-i x} \sin (x) / x$. The equations are written in scaled form and describe the amplitudes of the fundamental $A_{1}$, second harmonic $A_{2}$, and parametric fields $A_{ \pm}$. We have introduced $\gamma=\gamma_{2} / \gamma_{1}$ and $v=v_{2} / v_{1}$ as the ratio of the loss rates and effective propagation velocities between the second harmonic and the fundamental, respectively, while $\tau$ $=\tau_{1} / \tau_{2}$ denotes the ratio of the round-trip propagation times. The loss rates are given by $\gamma_{1}=\left(T+\mathcal{L}_{1}\right) / 2 \tau_{1}, \gamma_{2}$ $=\mathcal{L}_{2} / 2 \tau_{2}$, where $T$ is the intensity transmission of the input coupler and $\mathcal{L}_{1}, \mathcal{L}_{2}$ are residual passive losses of the fundamental and second harmonic during one round trip. All transmission and loss parameters are assumed small compared to unity to ensure the validity of the mean-field model. The propagation round-trip times are $\tau_{j}=2\left(L_{j}+n L_{c}\right) / c, j=1,2$, where $n$ is the refractive index of the nonlinear crystal and $c$ is the speed of light in vacuum, while the effective propagation velocities are given by $v_{j}=\left(L_{j}+L_{c} / n\right) / \tau_{j}, j=1,2$.

The detuning parameters $\Delta_{j}=\left(\omega_{j}-\omega_{j, c}\right) / \gamma_{1}, j=1,2,+$, - measure the difference between the optical frequency and the nearest cavity resonance frequency normalized to the loss rate of the fundamental. We will study a situation where $\Delta_{1}$ and $\Delta_{2}$ are free parameters that can be selected experimentally using, e.g., pump-laser frequency and cavity length, although other tuning control parameters are possible [10]. We implicitly assume that the actual detuning of the parametric fields is selected by the oscillator in order to maximize the parametric gain. When the frequency shift of the parametric fields with respect to the fundamental frequency is sufficiently small energy conservation written as $\omega_{+}+\omega_{-}$ $=2 \omega_{1}$ implies a slaving of the parametric detunings to the fundamental according to

$$
\Delta_{+}+\Delta_{-} \simeq 2 \Delta_{1}
$$

As discussed in the Appendix, Eq. (2) is a good approximation for parametric frequency shifts of order ten cavity free spectral ranges, and we will assume Eq. (2) to be valid in this 
paper. For larger parametric frequency shifts the parametric detunings may not satisfy Eq. (2). Analysis of that situation lies outside the scope of the present paper.

In addition to the slaving of the parametric detunings we will, without loss of generality, assume that $\Delta_{+}=\Delta_{-}$for the following reason. Making the transformations $A_{+} \rightarrow A_{+} e^{i \delta t}$, $A_{-} \rightarrow A_{-} e^{-i \delta t}$, leaves Eqs. (1) unchanged apart from renormalization of the parametric detunings as $\Delta_{+} \rightarrow \Delta_{+}-\delta$ and $\Delta_{-} \rightarrow \Delta_{-}+\delta$. Assume that we have unequal detunings with $\Delta_{+}=\Delta_{-}+\epsilon$. Then choosing $\delta=\epsilon / 2$ equalizes the renormalized $\Delta_{ \pm}$. We will therefore assume that a frequency shift $\delta$ has been applied such that $\Delta_{+}=\Delta_{-}$and using Eq. (2) then gives $\Delta_{+}=\Delta_{-}=\Delta_{1}$, which will be used in the rest of this paper.

The scaling used in Eqs. (1) is important in order to connect to the physical parameters. The electric-field amplitudes have been defined as $A_{j}=2 \kappa L_{c} \mathcal{E}_{j}^{(0)} /\left(\gamma_{1} \tau_{1} \sqrt{n}\right), j=1,2,+$, -, where $\mathcal{E}_{j}^{(0)}$ is the amplitude of the electric field in vacuum and $\kappa=\omega_{1} d_{\text {eff }} /(n c)$ is the strength of the nonlinear coupling, with $\omega_{1}$ the frequency of the fundamental field and $d_{\text {eff }}$ the effective nonlinearity coefficient. The time and space coordinates have been scaled according to the transformations $\gamma_{1} t \rightarrow t,\left(\sqrt{k_{1} \gamma_{1} / v_{1}}\right) \mathbf{r} \rightarrow \mathbf{r}$, where $k_{1}$ is the wave number of the fundamental in vacuum. The fundamental pump field has been scaled as $E=2 \eta \kappa L_{c} \mathcal{E}_{\text {pump }}^{(0)} /\left(\gamma_{1}^{2} \tau_{1}^{2} \sqrt{n}\right)$, where $\mathcal{E}_{\text {pump }}^{(0)}$ is the physical electric field in vacuum that can be chosen real due to the free choice of the absolute phase. $\eta$ $=\sqrt{T}$ is the input coupling efficiency of the pump field. Finally, $\xi=\Delta k L_{c}, \quad \widetilde{\xi}=\Delta \widetilde{k} L_{c}$ are the dimensionless phasemismatch parameters in the second-harmonic and parametric processes, respectively, where $\Delta k=2 k_{1}-k_{2}$ and $\Delta \tilde{k}=k_{+}$ $+k_{-}-k_{2}$.

In Eqs. (1) the parametric fields have been assumed to propagate the same distance and experience the same passive losses as the fundamental field. This is a very good assumption since phase-matching bandwidth restrictions imply that the parametric pairs are created with a frequency close to the fundamental. Furthermore, their frequencies will be close to a cavity resonance positioned an integer multiple of the cavity free spectral range away from the fundamental resonance.

\section{STABILITY ANALYSIS}

In this section we analyze the stability of the homogeneous solutions. The first subsection will concern the case where the system is below the parametric oscillation threshold where the parametric fields are zero. In this case instabilities seen in a pure SHG system are encountered. Furthermore, a stability analysis of the vanishing parametric solutions allows identification of the parametric oscillation threshold. The second subsection concerns the IPOPO above threshold for the parametric process. Exact solutions for the off-axis emitted parametric fields are derived and their stability is tested leading to secondary instabilities.

\section{A. Ground-state solutions below parametric threshold}

Below the parametric threshold, the homogeneous solutions for the parametric fields are $A_{+}^{0}=A_{-}^{0}=0$. The corre- sponding homogeneous solutions for the fundamental and second harmonic can be found from Eqs. (1) as a straightforward generalization of the expressions given in [5] to include phase-mismatch and different propagation distances between the fundamental and second harmonic. We obtain the following expressions

$$
\begin{aligned}
& {\left[\frac{\tau^{2}|f(\xi)|^{4}}{\gamma^{2}+\Delta_{2}^{2}}\left|A_{1}^{0}\right|^{4}+2 \frac{\gamma-\Delta_{1} \Delta_{2}}{\gamma^{2}+\Delta_{2}^{2}} \tau|f(\xi)|^{2}\left|A_{1}^{0}\right|^{2}+1+\Delta_{1}^{2}\right]} \\
& \times\left|A_{1}^{0}\right|^{2}=E^{2} \\
& \left|A_{2}^{0}\right|=\frac{\tau|f(\xi)|}{\sqrt{\gamma^{2}+\Delta_{2}^{2}}}\left|A_{1}^{0}\right|^{2} .
\end{aligned}
$$

A linear stability analysis is performed by perturbing the homogeneous solutions according to

$$
\begin{aligned}
& A_{1}=A_{1}^{0}+a_{1} \exp \left(\lambda t+i \mathbf{k}_{\perp} \cdot \mathbf{r}\right)+b_{1} \exp \left(\lambda * t-i \mathbf{k}_{\perp} \cdot \mathbf{r}\right), \\
& A_{2}=A_{2}^{0}+a_{2} \exp \left(\lambda t+i \mathbf{k}_{\perp} \cdot \mathbf{r}\right)+b_{2} \exp \left(\lambda * t-i \mathbf{k}_{\perp} \cdot \mathbf{r}\right), \\
& A_{ \pm}=a_{ \pm} \exp \left(\tilde{\lambda} t+i \tilde{\mathbf{k}}_{\perp} \cdot \mathbf{r}\right)+b_{ \pm} \exp \left(\tilde{\lambda} * t-i \tilde{\mathbf{k}}_{\perp} \cdot \mathbf{r}\right) .
\end{aligned}
$$

We note that the stability analysis for the parametric fields determines the threshold for onset of the parametric oscillations. The problem factorizes into two quartic characteristic polynomials in the two independent eigenvalues $\lambda$ and $\tilde{\lambda}$

$$
\begin{aligned}
& {\left[\lambda^{4}+2(1+\gamma) \lambda^{3}+a_{2}\left(k_{\perp}^{2}\right) \lambda^{2}+a_{1}\left(k_{\perp}^{2}\right) \lambda+a_{0}\left(k_{\perp}^{2}\right)\right]} \\
& \quad \times\left[\widetilde{\lambda}^{2}+2 \widetilde{\lambda}+1+\left(\widetilde{k}_{\perp}^{2}-\Delta_{1}\right)^{2}-|f(\widetilde{\xi})|^{2}\left|A_{2}^{0}\right|^{2}\right]^{2}=0,
\end{aligned}
$$

where the coefficients are given by

$$
\begin{aligned}
a_{0}\left(k_{\perp}^{2}\right)= & 4 \tau|f(\xi)|^{2}\left|A_{1}^{0}\right|^{2}\left(\tau|f(\xi)|^{2}\left|A_{1}^{0}\right|^{2}+\gamma-\delta_{1} \delta_{2}\right) \\
& +\left(\gamma^{2}+\delta_{2}^{2}\right)\left(1+\delta_{1}^{2}-|f(\xi)|^{2}\left|A_{2}^{0}\right|^{2}\right), \\
a_{1}\left(k_{\perp}^{2}\right)= & 4(1+\gamma) \tau|f(\xi)|^{2}\left|A_{1}^{0}\right|^{2}+2 \gamma\left(1+\gamma+\delta_{1}^{2}\right)+2 \delta_{2}^{2} \\
& -2 \gamma|f(\xi)|^{2}\left|A_{2}^{0}\right|^{2}, \\
a_{2}\left(k_{\perp}^{2}\right)= & |f(\xi)|^{2}\left(4 \tau\left|A_{1}^{0}\right|^{2}-\left|A_{2}^{0}\right|^{2}\right)+1+\gamma(4+\gamma)+\delta_{1}^{2}+\delta_{2}^{2},
\end{aligned}
$$

and all the dependence on the transverse wave number $k_{\perp}$ is in the generalized detuning coefficients

$$
\begin{aligned}
& \delta_{1}=\Delta_{1}-k_{\perp}^{2}, \\
& \delta_{2}=\Delta_{2}-\frac{1}{2} v k_{\perp}^{2} .
\end{aligned}
$$

One class of instabilities is obtained by solving $\operatorname{Re}(\lambda)$ $=0$. They appear in pure SHG, where the parametric fields 
are not excited, and the four different types of instabilities were investigated first by Etrich et al. $[4,5]$ and briefly reviewed below.

In the case of homogeneous perturbations without any spatial modulation $\left(k_{\perp}=0\right)$ bistability occurs and the threshold is obtained by solving $a_{0}\left(k_{\perp}^{2}=0\right)=0$. In the bistability regime localized structures can be found numerically. By solving $\lambda=i \omega_{c}$ in the characteristic equation, a Hopf instability is obtained, which implies time oscillating solutions with a frequency given by

$$
\omega_{c}=\sqrt{\frac{a_{1}\left(k_{\perp}^{2}\right)}{2(1+\gamma)}} .
$$

This phenomenon, known as self-pulsing, was first predicted by Drummond et al. [18]. Transverse instabilities are found by solving $a_{0}\left(k_{\perp}^{2}\right)=0$ allowing for $k_{\perp}$ to be different from zero. The most unstable transverse wave-vector component $k_{c}$ is located by furthermore requiring $\partial \operatorname{Re}(\lambda) / \partial k_{\perp}=0$, which is equivalent to $\partial a_{0}\left(k_{\perp}^{2}\right) / \partial k_{\perp}^{2}=0$. By differentiating Eq. (6a) a cubic equation in $k_{\perp}^{2}$ is obtained that can be solved to find $k_{c}$. Finally, also an oscillatory transverse instability can occur with the oscillation frequency $\omega_{c}$ given by Eq. (8) and with the transverse wave number and threshold obtained by solving the equations

$$
\begin{gathered}
\omega_{c}^{4}-a_{2}\left(k_{\perp}^{2}\right) \omega_{c}^{2}+a_{0}\left(k_{\perp}^{2}\right)=0, \\
\frac{\partial a_{2}\left(k_{\perp}^{2}\right)}{\partial k_{\perp}^{2}}+\left(\frac{a_{2}\left(k_{\perp}^{2}\right)}{a_{1}\left(k_{\perp}^{2}\right)}-\frac{1}{1+\gamma}\right) \frac{\partial a_{1}\left(k_{\perp}^{2}\right)}{\partial k_{\perp}^{2}}-\frac{1}{\omega_{c}^{2}} \frac{\partial a_{0}\left(k_{\perp}^{2}\right)}{\partial k_{\perp}^{2}}=0 .
\end{gathered}
$$

From the expressions in this paragraph the spatial scale $k_{c}$, oscillating frequency $\omega_{c}$, as well as the threshold amplitudes $E,\left|A_{1}\right|,\left|A_{2}\right|$ for the various instabilities can be found. In this paper we are in particular concerned with how these SHG instabilities are modified due to the competing parametric process in the IPOPO. The detailed analysis of this problem is given in Sec. IV.

A fundamentally different instability is the oscillation of the parametric process investigated by substituting $\widetilde{\lambda}_{R e}=0$ in the characteristic polynomial of Eq. (5) as treated in Ref. [9]. The threshold amplitude for this instability is given by

$$
\left|A_{1, p}^{0}\right|^{2}=\frac{\sqrt{\gamma^{2}+\Delta_{2}^{2}}}{\tau|f(\xi)||f(\tilde{\xi})|} \times \begin{cases}1 & \text { if } \Delta_{1}>0 \\ \sqrt{1+\Delta_{1}^{2}} & \text { if } \Delta_{1}<0\end{cases}
$$

The parametric pairs are emitted off axis with $\widetilde{k}_{c}=\sqrt{\Delta_{1}}$ for $\Delta_{1}>0$ and on axis $\widetilde{k}_{c}=0$ for $\Delta_{1}<0$. A more elaborate description of this parametric instability is given in the next section where exact solutions above threshold for the parametric process are found.

\section{B. Exact solutions above parametric threshold}

The exact analytical solutions are homogeneous plane waves for the fundamental and second harmonic, and off- axis emitted traveling waves for the parametric fields, corresponding to the following ansatz

$$
\begin{gathered}
A_{1}=\bar{A}_{1}, \\
A_{2}=\bar{A}_{2}, \\
A_{ \pm}=\bar{A}_{ \pm} \exp \left[ \pm i\left(\tilde{\mathbf{k}}_{\perp} \cdot \mathbf{r}+\tilde{\omega} t\right)\right] .
\end{gathered}
$$

After substitution into Eqs. (1), and using $\Delta_{+}=\Delta_{-}=\Delta_{1}$, we obtain

$$
\begin{gathered}
\left(1-i \Delta_{1}\right) \bar{A}_{1}=f(\xi) \bar{A}_{1}^{*} \bar{A}_{2}+E, \\
\left(\gamma-i \Delta_{2}\right) \bar{A}_{2}=-\tau f^{*}(\xi) \bar{A}_{1}^{2}-2 \tau f^{*}(\widetilde{\xi}) \bar{A}_{+} \bar{A}_{-}, \\
{\left[1+i\left(\tilde{\omega}+\widetilde{k}_{\perp}^{2}-\Delta_{1}\right)\right] \bar{A}_{+}=f(\widetilde{\xi}) \bar{A}_{2} \bar{A}_{-}^{*},} \\
{\left[1+i\left(\tilde{\omega}-\widetilde{k}_{\perp}^{2}+\Delta_{1}\right)\right] \bar{A}_{-}^{*}=f^{*}(\widetilde{\xi}) \bar{A}_{2}^{*} \bar{A}_{+} .}
\end{gathered}
$$

The existence of nontrivial solutions for the parametric fields requires $\tilde{\omega}=0$ and we have $\left|\bar{A}_{+}\right|=\left|\bar{A}_{-}\right| \equiv|\bar{A}|$. The amplitudes of the exact solutions are obtained by solving

$$
\begin{gathered}
\left|\bar{A}_{1}\right|^{2}=\zeta_{1}\left(E^{2}\right)-\zeta_{2}|\bar{A}|^{2}, \\
\left|\bar{A}_{2}\right|^{2}=\frac{\delta\left(\widetilde{k}_{\perp}^{2}\right)}{|f(\widetilde{\xi})|^{2}}, \\
|\bar{A}|^{4}+\zeta_{3}|\bar{A}|^{2}+\zeta_{4}=\frac{1}{4} \frac{|f(\xi)|^{2}}{|f(\widetilde{\xi})|^{2}}\left|\bar{A}_{1}\right|^{4},
\end{gathered}
$$

where we have introduced the coefficients

$$
\begin{gathered}
\zeta_{1}\left(E^{2}\right)=\frac{\tau|f(\widetilde{\xi})|^{2} E^{2}-2 \delta\left(\widetilde{k}_{\perp}^{2}\right)\left(\gamma-\Delta_{1} \Delta_{2}\right)}{\tau|f(\widetilde{\xi})|^{2}\left(1+\Delta_{1}^{2}\right)+\tau|f(\xi)|^{2} \delta\left(\widetilde{k}_{\perp}^{2}\right)}, \\
\zeta_{2}=\frac{4|f(\widetilde{\xi})|^{2} \sqrt{\delta\left(\widetilde{k}_{\perp}^{2}\right)}\left[\cos \phi\left(\widetilde{k}_{\perp}^{2}\right)+\Delta_{1} \sin \phi\left(\widetilde{k}_{\perp}^{2}\right)\right]}{|f(\widetilde{\xi})|^{2}\left(1+\Delta_{1}^{2}\right)+|f(\xi)|^{2} \delta\left(\widetilde{k}_{\perp}^{2}\right)}, \\
\zeta_{3}=\frac{\sqrt{\delta\left(\widetilde{k}_{\perp}^{2}\right)}\left[\gamma \cos \phi\left(\widetilde{k}_{\perp}^{2}\right)-\Delta_{2} \sin \phi\left(\widetilde{k}_{\perp}^{2}\right)\right]}{\tau|f(\widetilde{\xi})|^{2}}, \\
\zeta_{4}=\frac{\delta\left(\widetilde{k}_{\perp}^{2}\right)\left(\gamma^{2}+\Delta_{2}^{2}\right)}{4 \tau^{2}|f(\widetilde{\xi})|^{4}},
\end{gathered}
$$

and all the dependence on the transverse wave number $\widetilde{k}_{\perp}^{2}$ is in the parameters

$$
\begin{gathered}
\delta\left(\widetilde{k}_{\perp}^{2}\right)=1+\left(\Delta_{1}-\widetilde{k}_{\perp}^{2}\right)^{2}, \\
\phi\left(\widetilde{k}_{\perp}^{2}\right)=\arctan \left(\Delta_{1}-\widetilde{k}_{\perp}^{2}\right) .
\end{gathered}
$$


These exact solutions above threshold of the parametric process were stated in Ref. [14] under simplified conditions of $v=\tau=1$ and equal phase-matching parameters $\xi=\widetilde{\xi}$. Relaxing on the latter condition is found to lead to new types of instabilities as investigated below.

In order to perform a stability analysis of the exact solutions also the phases must be found. We introduce the following notation:

$$
\bar{A}_{j}=\left|\bar{A}_{j}\right| e^{i \phi_{\bar{A}_{j}}},
$$

$j=1,2,+,-$. It turns out that only the sum of the parametric phases can be calculated from the Eqs. (12). We will assume symmetric conditions with $\phi_{\bar{A}_{+}}=\phi_{\bar{A}_{-}} \equiv \phi_{\bar{A}} / 2$. The phases can be found by solving

$$
\begin{aligned}
\phi_{\bar{A}_{1}}= & \arg \left[\tau\left(1+i \Delta_{1}\right)\left|\bar{A}_{1}\right|^{2}+\left(\gamma-i \Delta_{2}\right)\left|\bar{A}_{2}\right|^{2}\right. \\
& \left.+2 \tau|f(\widetilde{\xi})|\left|\bar{A}_{2}\right||\bar{A}|^{2} e^{i \phi\left(\widetilde{k}_{\perp}^{2}\right)}\right], \\
\phi_{\bar{A}_{2}}= & \arg \left[-\left(\gamma+i \Delta_{2}\right)\left|\bar{A}_{2}\right|-2 \tau|f(\widetilde{\xi})||\bar{A}|^{2}\right. \\
& \left.\times e^{-i \phi\left(\widetilde{k}_{\perp}^{2}\right)}\right]+2 \phi_{\bar{A}_{1}}-\phi_{f}, \\
\phi_{\bar{A}}= & \phi_{\bar{A}_{2}}+\phi_{\tilde{f}}+\phi\left(\widetilde{k}_{\perp}^{2}\right),
\end{aligned}
$$

where $\phi_{f}=\arg [f(\xi)]$ and $\phi_{\tilde{f}}=\arg [f(\widetilde{\xi})]$. Note that the freedom in choice of the parametric phases implies that their difference is subject to a diffusive process [19]. Numerical simulations in regimes where the exact solutions are stable, support the presence of such a phase diffusive process. It is observed that different initial noise levels and noise values result in different end results for the parametric phases, although the sum of these is in correspondence with the value calculated with Eq. (17c).

Bistability in the parametric solutions occurs when Eq. (13c) has two physical solutions for a given value of $E$. It arises when the ground-state solutions $A_{ \pm}=0$ become unstable through a subcritical bifurcation, and thus corresponds to bistability between the parametric solutions below and above threshold. This turns out to happen only for negative fundamental detuning where the parametric fields are emitted on axis, $\widetilde{k}_{\perp}=0$. Necessary conditions for the parametric bistability to exist include unequal phase mismatch of the two competing processes $(\xi \neq \widetilde{\xi})$ as well as

$$
\frac{|f(\xi)|^{2}+|f(\widetilde{\xi})|^{2}}{2|f(\xi)||f(\widetilde{\xi})|}\left(\Delta_{1} \Delta_{2}-\gamma\right)>\sqrt{\left(1+\Delta_{1}^{2}\right)\left(\gamma^{2}+\Delta_{2}\right)},
$$

which implies $\Delta_{1} \Delta_{2}>\gamma$. The bistability region is $E_{1}<E$ $<E_{p}$ with the boundary values given by

$$
\begin{aligned}
E_{p}^{2}= & \frac{1+\Delta_{1}^{2}}{\tau|f(\tilde{\xi})|^{2}}\left(\frac{|f(\xi)|^{2}+|f(\widetilde{\xi})|^{2}}{|f(\xi)||f(\tilde{\xi})|}\right. \\
& \left.\times \sqrt{\left(1+\Delta_{1}^{2}\right)\left(\gamma^{2}+\Delta_{2}^{2}\right)}+2\left(\gamma-\Delta_{1} \Delta_{2}\right)\right),
\end{aligned}
$$

$$
E_{1}^{2}=\frac{\left.|| f(\xi)\right|^{2}-|f(\widetilde{\xi})|^{2} \mid}{\tau|f(\xi)||f(\widetilde{\xi})|^{3}}\left(1+\Delta_{1}^{2}\right)\left|\Delta_{2}+\gamma \Delta_{1}\right|,
$$

where $E_{p}$ is the parametric oscillation threshold. Thus, the IPOPO contains two distinct types of bistabilities that can be attributed to the SHG and OPO processes, respectively. However, while the SHG bistability is found to behave in a similar way as in a pure SHG system, the parametric bistability is found to lead to new effects compared to the externally pumped OPO, as will be explored in Sec. IV B. Studies of bistability and cavity solitons in the ordinary pumped OPO have been performed both in the degenerate [20-22] and nondegenerate configurations [3,23-25].

The exact parametric solutions can be tested by a stability analysis. The solutions are perturbed according to

$$
\begin{aligned}
A_{j}= & \bar{A}_{j}+a_{j} \exp (\Lambda t+i \mathbf{K} \cdot \mathbf{r})+b_{j} \exp \left(\Lambda^{*} t-i \mathbf{K} \cdot \mathbf{r}\right), \\
A_{ \pm}= & \exp \left( \pm i \widetilde{\mathbf{k}}_{\perp} \cdot \mathbf{r}\right)\left[\bar{A}_{ \pm}+a_{ \pm} \exp (\Lambda t+i \mathbf{K} \cdot \mathbf{r})+b_{ \pm}\right. \\
& \left.\times \exp \left(\Lambda^{*} t-i \mathbf{K} \cdot \mathbf{r}\right)\right],
\end{aligned}
$$

$j=1,2$. This analysis is similar to the one described in Sec. III A except that it is further complicated by the nonvanishing amplitudes of the parametric fields leading to an $8 \times 8$ matrix problem for computation of the eigenvalues. Equations (11) constitute a continuous family of exact solutions by varying the wave number $\widetilde{k}_{\perp}$. However, at threshold $\widetilde{k}_{c}$, obtained from the parametric threshold analysis, is selected. The numerical analysis indicates that the solution excited at threshold also prevails further above threshold. The same conclusion is reached by investigating growth rates above threshold that are found to be peaked at $\widetilde{k}_{c}$, and hence we let $\widetilde{k}_{\perp}=\widetilde{k}_{c}$ in Eqs. (20). For $\Delta_{1}>0$, the direction of the wave vector $\mathbf{K}$ introduced in the transverse perturbations becomes important. It can be directed either parallel or perpendicular to the wave vector $\widetilde{\mathbf{k}}_{c}$ of the exact off axis solution, corresponding to Eckhaus and zig-zag instabilities, respectively. For $\Delta_{1}<0$, the parametric fields are emitted on axis and therefore the direction of $\mathbf{K}$ is unimportant. The results of this stability analysis are given in the following section.

\section{INSTABILITIES IN THE IPOPO}

In this section results from the linear stability analysis are presented, and the formation of patterns above instability threshold is investigated numerically using a split-step routine previously discussed in Ref. [7]. The results illustrate two fundamentally different situations. First we demonstrate that the SHG temporal and spatial instabilities can be observed also in the presence of the competing parametric process, which occurs for parameters where the SHG instability thresholds are below the parametric threshold. In the opposite case, a stability analysis of the exact solutions in Eqs. (13) predicts quenching of the SHG patterns. In certain regions, these exact solutions may destabilize through a secondary instability that recently was shown to lead to forma- 
tion of novel intensity spiral structures [14]. In this section other examples of secondary instabilities are given including parametric bistability and self-pulsing.

In previous work the extra degrees of freedom associated with phase mismatch of the conversion processes have not been considered. Introducing nonzero but equal phase mismatches $(\xi=\widetilde{\xi} \neq 0)$ leads to an identical term $f(\xi)$ in front of all nonlinear terms in Eqs. (1) which can be scaled away.

Hence, $\xi=\widetilde{\xi} \neq 0$ does not lead to any new physical effects but only to an increase in the thresholds for the instabilities. This is in contrast to the singly resonant second-harmonic generation (SRSHG) configuration where phase mismatch was found to be an important tuning parameter [7]. This difference arises since the axial variation of the secondharmonic field in doubly resonsant SHG is averaged out in the well-established mean-field description, which is not appropriate for the second harmonic in SRSHG.

Introducing different phase mismatches for the two $\chi^{(2)}$ processes $(\xi \neq \widetilde{\xi})$ allows independent tuning of the SHG instabilities and the parametric threshold, since the former are independent of $\widetilde{\xi}$ while the latter depend on both $\xi$ and $\widetilde{\xi}$. This can, e.g., be used to move the parametric instability above the SHG instabilities in order to avoid undesired quenching of the pattern formation. Furthermore, it turns out that new parametric instabilities can arise that are not present in the equally phase-matched case, as will be described in Sec. IV B. However, in an experimental context, it is worth emphasizing that while the phase-matching parameter $\xi$ for the SHG process can be controlled, the parametric pairs will be emitted with frequencies that lower the parametric threshold. From Eq. (10) this is seen to lead to $\widetilde{\xi}=0$, which will be assumed to be the case in this paper. In certain situations this may be a too simplistic description since also the requirement of a nearby cavity resonance contributes to the selection of the parametric frequencies, as was discussed extensively in Ref. [10].

Unless otherwise stated, in this section we will assume ideal phase matching $\xi=\widetilde{\xi}=0$ and equal propagation distances for the fundamental and second harmonic $\tau=v=1$. The significance of unequal propagation distances in an experimental context is discussed in Sec. V.

\section{A. SHG instabilities}

In the ideally phase-matched case two distinct parameter regions exist depending on the sign of the fundamental detuning $\Delta_{1}$. For positive $\Delta_{1}$, the parametric instability is lowest in the system and dominates the behavior as demonstrated in Ref. [9]. Here the robustness of the parametric off-axis solutions was seen numerically and found to lead to quenching of pattern formation. Although the parametric fields are emitted off axis in the cavity, the process is not found to lead to any spatial intensity modulation since the nondegenerate parametric fields cannot interfere. In addition to the numerical studies, the parametric quenching of SHG instabilities can be investigated analytically by the stability analysis of the exact parametric solutions as was outlined in Sec. III B. The parametric solutions appear always to be stable both for perturbations parallel and perpendicular to $\widetilde{\mathbf{k}}_{c}$, thus indicating a complete quenching of pattern formation for positive detuning.

Introducing unequal phase mismatches in the competing processes $(\xi \neq \widetilde{\xi})$, the parametric threshold may be shifted above the SHG thresholds. In this way the SHG instabilities can be excited also for $\Delta_{1}>0$ when using a pump level between the SHG and parametric thresholds. Increasing $E$ above the parametric threshold, however, quenching is found both in the stability analysis and numerically, and it is again due to the off-axis emitted parametric fields. Quenching from the parametric process thus seems to be a generic property in the IPOPO for positive fundamental detuning.

For negative fundamental detuning $\Delta_{1}<0$, parameter regions exist where all types of SHG instabilities have lowest threshold, i.e., they can be excited. Increasing the pump above the parametric threshold, the SHG instabilities can prevail in this case leading to the formation of the same modulated structures also in the parametric fields. An example of hexagonal structures in all four fields can be found in Ref. [9], and furthermore traveling waves have been observed. In some cases the SHG instabilities may be changed subsequently when increasing the pump above the parametric threshold as well. Such an example is described in the next section.

Also the temporal self-pulsing instability can be excited for $\Delta_{1}<0$. This is in contrast to the resonant case treated in Ref. [11] where the parametric process was found to quench self-pulsing completely. The presence of self-pulsing in the detuned system was suggested in [26] and is here demonstrated explicitly. For the parameters in Fig. 2, close to the self-pulsing threshold $\left(E_{\mathrm{SP}} \simeq 10.4\right)$ a single-frequency oscillation is observed with a frequency in agreement with the linear stability result $\omega_{c}$. Further, above threshold, the parametric threshold $\left(E_{p} \simeq 10.9\right)$ is crossed, and self-pulsing in all four fields is obtained as shown in Fig. 2. The primary frequency of the oscillations is $\omega=3.39$, in reasonable agreement with the frequency predicted by the linear stability threshold analysis of $\omega_{c}=3.17$. We observe the presence of period doubling as most clearly seen in the fundamental field. Such period doubling was previously found in SHG by Savage and Walls [27] to be a prerequisite for chaotic oscillations.

The presence of bistability in SHG was extensively discussed in Ref. [4]. In that case cavity solitons can be formed as a consequence of bistability between homogeneous stable and modulationally unstable solutions. This SHG bistability is also found in the IPOPO in the case where both the fundamental and second harmonic have sufficiently large negative detunings. In this region, excitation of cavity solitons in all four fields is possible but will not be discussed further here.

\section{B. Secondary parametric instabilities}

Also for negative fundamental detuning the parametric threshold can be the lowest instability in the system for certain parameters. Hence, the exact parametric solution in Eqs. (11) can be excited, where in that case the parametric fields 

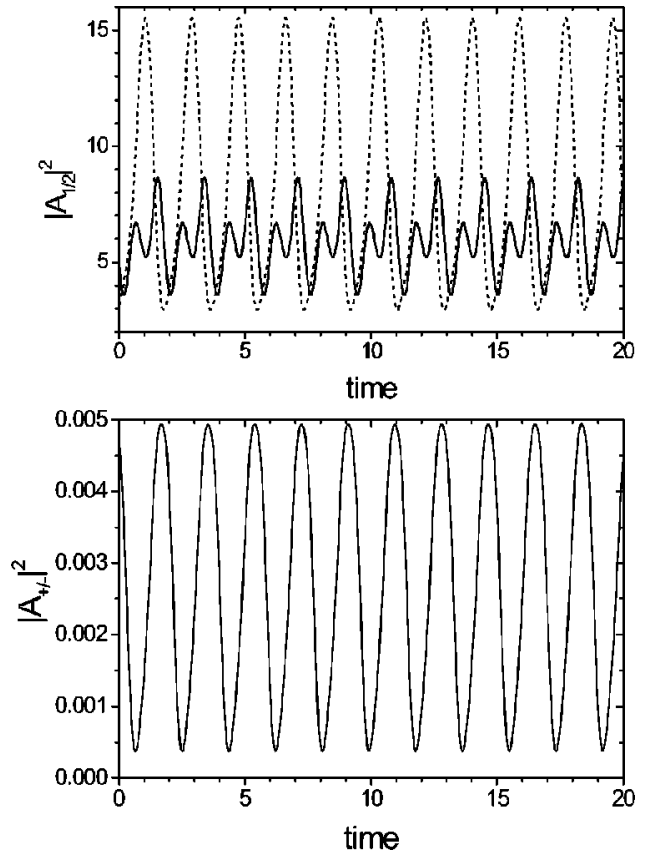

FIG. 2. Self-pulsing solution for all four cavity fields with $\Delta_{1}$ $=-2.5, \Delta_{2}=1.2$, and $\gamma=1$. The pump level is $E=11.7$. The plots display the scaled intensity in the four beams as a function of time. The upper plot shows the fundamental (solid) and second-harmonic (dashed) intensities, while the lower plot shows the parametric intensities.

are emitted on axis with $\widetilde{k}_{\perp}=0$. These solutions can become unstable when increasing $E$ due to the presence of secondary instabilities in the system. This is in contrast to the off-axis solutions discussed in the previous section that were always linearly stable. Spatiotemporal pattern formation from this secondary instability was investigated in Ref. [14]. There the formation of traveling roll patterns was observed that could destabilize further to form intensity spiral structures constituting a novel type of nonlinear phenomenon in optics.

When the parametric threshold is above an SHG pattern threshold the assumption of spatially homogeneous fundamental and second-harmonic fields may not be accurate. However, if the modulation from the SHG instability is weak the ansatz given by Eqs. (11) may still be a reasonable approximation. This allows an analysis of the effect of the parametric process, also in this case, as discussed in the following.

Figure 3 shows growth rates found from stability analysis of the parametric solutions. The parameters in this figure correspond to the case where the SHG traveling-wave threshold is lowest, $E_{\mathrm{TW}} \simeq 14.3$, while the SHG self-pulsing threshold is $E_{\mathrm{SP}} \simeq 15.3$, and the parametric threshold is $E_{p} \simeq 15.7$. The full curve in Fig. 3 shows the growth rate just above $E_{p}$. The system is seen to be unstable towards perturbations with both $K_{c}=0$ and $K_{c} \simeq 0.7$, corresponding to self-pulsing and traveling-wave instabilities, respectively, since both have $\operatorname{Im}\left[\Lambda_{\max }\left(K_{c}\right)\right] \neq 0$. They are remains of the SHG instabilities present below $E_{p}$. As $E$ is increased slightly to $E$ $=16.2$ (dashed curve), the self-pulsing is quenched while the traveling-wave instability becomes quenched after increasing

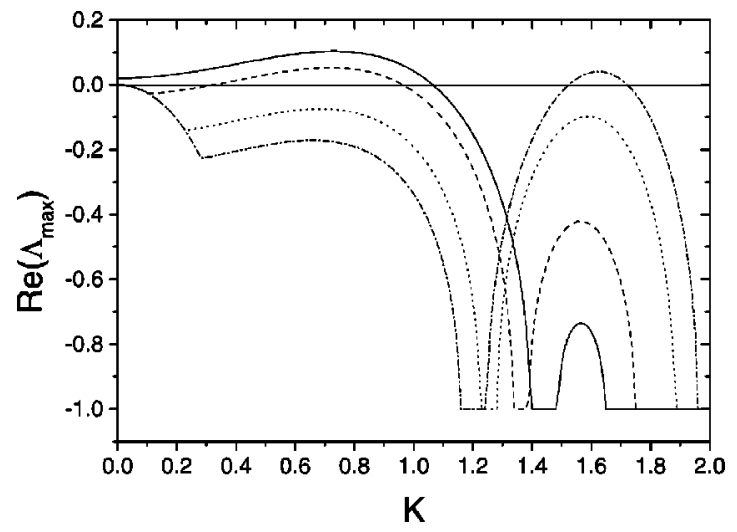

FIG. 3. Growth rates as a function of the transverse perturbation wave number $K$ for the parameters $\Delta_{1}=-2.5, \Delta_{2}=3.0$, and $\gamma=1$. The pump level is $E=15.8$ (full curve), $E=16.2$ (dashed curve), $E=17.2$ (dotted curve), and $E=18.0$ (dash-dotted curve).

the pump to $E=17.2$ (dotted curve). However, another traveling-wave instability is found at a pump level of $E$ $=18.0$ (dash-dotted curve), with a spatial instability wave number $K_{c} \simeq 1.65$.

Numerical simulations confirm the above scenario. At $E$ $=15.8$ the SHG traveling waves are found with a transverse wave number close to $K \simeq 0.7$. At $E=18.0$ the traveling waves in Fig. 4 are obtained, which has a transverse wave vector close to $K_{c} \simeq 1.65$ that was predicted from the dashdotted curve in Fig. 3. This clearly shows that the traveling waves in Fig. 4 are due to a secondary parametric instability, in this case different from the SHG instabilities.

Allowing the two competing nonlinear processes to be unequally phase matched $(\xi \neq \widetilde{\xi})$ and keeping the detunings sufficiently negative, bistability from the parametric process can occur, as was shown in Sec. III B. Figure 5 shows a bifurcation diagram for $\xi=2.5, \widetilde{\xi}=0, \Delta_{1}=-7$, and $\gamma=2$, where the parametric bistability is seen to set in at $\Delta_{2} \simeq$ -1.4 . The phase-mismatch parameter for the SHG process is

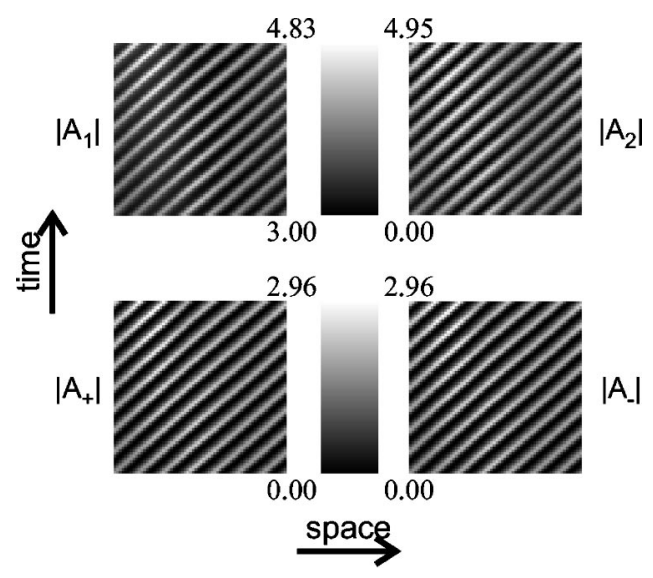

FIG. 4. Space-time plot showing a traveling-wave pattern due to a secondary instability with parameters as in Fig. 3. The pictures show the temporal evolution of a cut through the transverse plane. The horizontal box size is 27.0 while the vertical time axis is 10 time units. The pump level is $E=18.0$. 


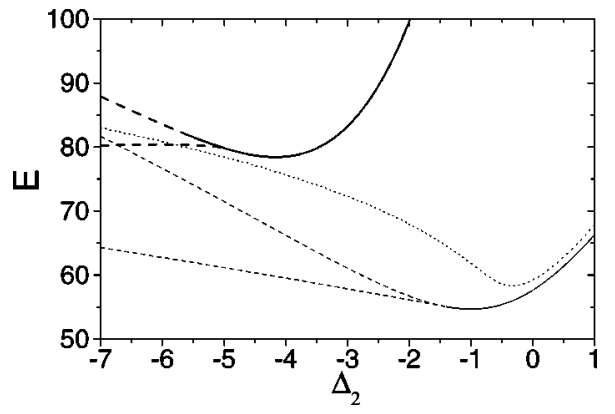

FIG. 5. Bifurcation diagram for $\Delta_{1}=-7, \gamma=2, \xi=2.5$, and $\widetilde{\xi}$ $=0$. The bold-solid line is the SHG transverse threshold while the bold-dashed lines are limit points of the SHG bistable area. The thin-solid line is the parametric threshold, the thin-dashed lines are limits of the parametric bistable area. Finally, the thin-dotted line is the self-pulsing secondary instability.

chosen sufficiently large such that the parametric threshold is below the SHG thresholds. For a fixed value of the secondharmonic detuning, $\Delta_{2}=-4$, the lower plot in Fig. 6 shows the characteristic bistability curve of the intracavity parametric intensity as a function of the pump $E$. At the oscillation threshold $\left(E_{p} \simeq 66.2\right.$ ) the ground-state parametric solution, $A_{ \pm}=0$ (solid curve), bifurcates subcritically into an unstable branch (dotted curve). This middle branch is linearly unstable and using Eq. (19b) is found to bifurcate at $E_{1}$ $\simeq 59.5$ into a modulationally unstable upper branch (dashed curve). The corresponding bistability curves for the fundamental and second harmonic are also displayed in Fig. 6. The fundamental bistability curve is seen to be inverted such that the modulational branch is below the homogeneous solution. The second harmonic, however, is clamped at a constant value above the parametric threshold as is also seen from Eq. (13b), which follows from the exact balancing of the two competing frequency conversion processes in steady state. This clamping was observed in Ref. [28].

The inverted bistability curve for the fundamental field promises formation of spatial structures consisting of holes in the homogeneous background. An example of such a honeycomb structure in the fundamental is shown in Fig. 7 for $E=68.0$ corresponding to a pump level above the bistability region. The modulated structures in the three other fields are ordinary hexagons in agreement with expectations from Fig. 6 . The average intensities are found to oscillate in time due to a self-pulsing instability in the system, which could not be predicted directly from the stability analysis.

Bistability between a homogeneous stable ground state and a modulationally unstable upper state can lead to formation of localized structures, also called cavity solitons. Unlike temporal solitons in, e.g., fiber optics, these solitons are a result of a careful preparation of the system. Using a localized address beam a small part of the system is set in the modulationally unstable upper state, while the rest of the system is maintained at the homogeneous background level. These states must be connected to each other by transitional kink type waves, also called switching waves [29], that can lock mutually to form solitonlike structures.
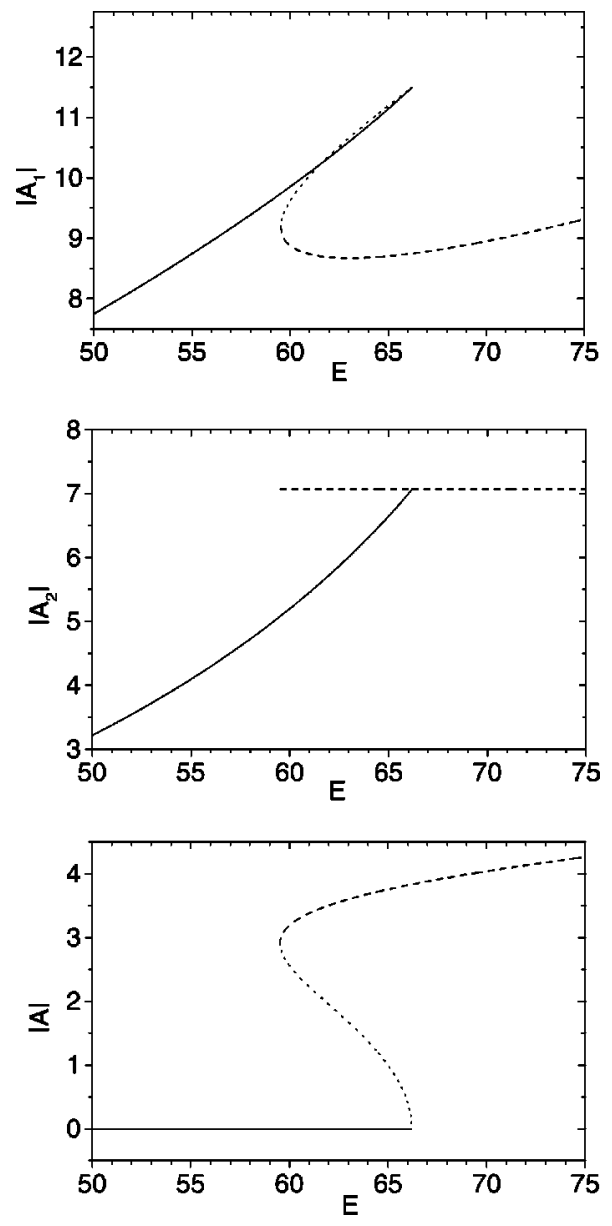

FIG. 6. Intracavity fields as a function of $E$. The full (dashed) curves are the solutions below (above) the parametric threshold while the dotted curves are linearly unstable homogeneous branches. The parameters are as in Fig. 5, and with $\Delta_{2}=-4$.

In order to investigate the formation of cavity solitons numerically, the system was prepared as follows. The localized Gaussian address beam had a pump value well above the upper limit of the bistable area. After 5 time units the address beam was switched off and the evolution of the fields was followed. For a pump value close to the bistable lower limit, $E=59.7$, it is possible to obtain stable cavity solitons, where an example is shown in Fig. 8. The fundamental soliton is a so-called gray soliton, since it constitutes a localized hole in the homogeneous background as a consequence of the inverted fundamental bistability curve. The second harmonic and parametric solitons are all bright solitons, and generally the solitons may be seen as residuals of the modulational structure of the upper branch, which in this case are the hexagons shown in Fig. 7. Note that cavity solitons may also be observed by pumping well above the bistability limits to obtain the hexagonal patterns in Fig. 7, and then slowly decreasing the pump level to enter the bistable area. The appearance of two-dimensional (2D) gray solitons is, to our knowledge, a new phenomenon in $\chi^{(2)}$ cavity interactions. However, 1D dark solitons have been reported in the OPO [30] due to a different mechanism based on the coexistence of two parametric homogeneous solutions with 

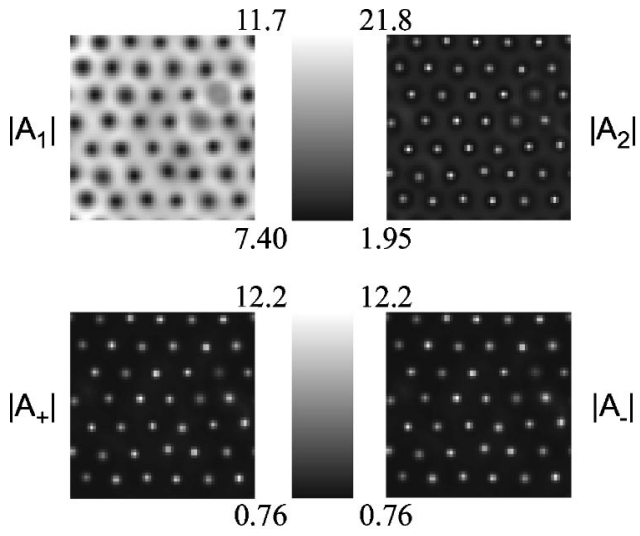

FIG. 7. Hexagons in the transverse plane originating from the modulationally unstable upper branch in Fig. 6. The fundamental field displays honeycomb hexagons, while the second-harmonic and parametric fields show ordinary hexagons. The window size is $21.4 \times 21.4$ and the parameters are as in Fig. 6 with $E=68.0$.

different phases. In 2D these solitons are manifested as dark stripes or domain walls.

Increasing the pump level, the modulational branch becomes Hopf unstable and self-pulsing solitons are observed. An example is shown in Fig. 9 where results from the first 20 time units of a simulation is shown, and after the address beam is turned off the soliton oscillates regularly. Similar oscillating solitons have been observed numerically in the nondegenerate OPO [31] (bright solitons) and in a cavity filled with a saturable absorber [32] (gray solitons).

The exact solutions above the parametric threshold may also destabilize through a homogeneous Hopf bifurcation corresponding to an instability to a perturbation with $K_{c}=0$ and $\Lambda= \pm i \Omega_{c}$. This self-pulsing secondary instability is also included in the bifurcation diagram in Fig. 5. For $\Delta_{2}$ $=0$ and with a pump close to the self-pulsing threshold, it is possible to obtain single-frequency oscillations with $\Omega$ $\simeq 1.90$ very close to the theoretical value of $\Omega_{c} \simeq 1.93$. These oscillations lead to similar self-pulsing intensity behavior in all four fields as shown in Fig. 2, however the mechanism is in this case different and due to a secondary instability. The
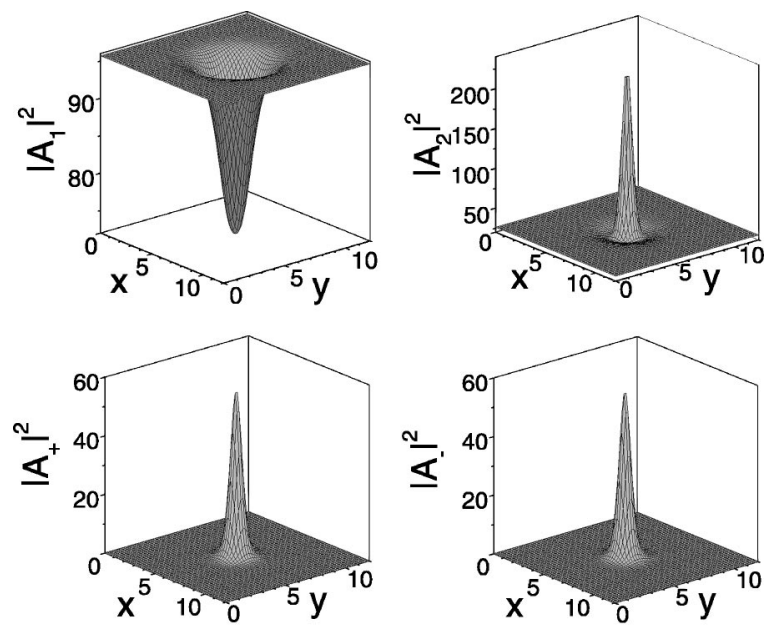

FIG. 8. Cavity solitons for $E=59.7$ and the parameters in Fig. 6 .

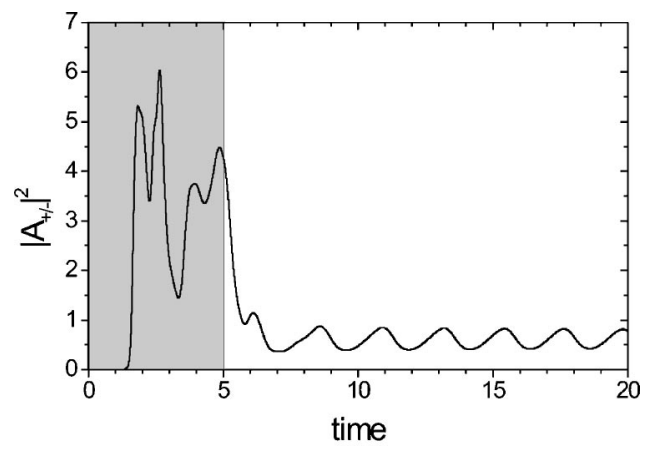

FIG. 9. Self-pulsing cavity solitons for $E=62.0$ and the parameters in Fig. 6. The plot shows the average intensity of the parametric fields as a function of time. The gray area indicates the first five time units of the simulation where the address beam is on with a value of $E=84.0$.

self-pulsing threshold is found to occur above the pump level where the oscillations of the hexagons and cavity solitons starts and could indicate the self-pulsing bifurcation is subcritical. However, more involved mechanisms may be responsible for the oscillations, as reported for nonlinear Kerr resonators [33], where the self-pulsing is found from destabilization of the modulated structure as opposed to the homogeneous solutions studied here.

\section{EXPERIMENTAL PARAMETERS}

The stability analysis presented in Sec. III was done in scaled parameters. In order to relate to parameters in a real experiment it is necessary to convert back to physical units. In the following we will calculate the thresholds for the different SHG instabilities and the corresponding spatial and temporal scales for relevant physical parameters.

From the scalings introduced in Sec. II B, we obtain

$$
\begin{gathered}
\tau=\frac{\tau_{1}}{\tau_{2}}=\frac{L_{1}+n L_{c}}{L_{2}+n L_{c}}, \\
\gamma=\frac{\gamma_{2}}{\gamma_{1}}=\frac{\mathcal{F}_{1}}{\mathcal{F}_{2}} \frac{L_{1}+n L_{c}}{L_{2}+n L_{c}}, \\
\Delta_{j}=\gamma_{1} \bar{\Delta}_{j}=\frac{\pi c}{2 \mathcal{F}_{1}} \frac{1}{L_{1}+n L_{c}} \bar{\Delta}_{j}, \\
v=\frac{v_{2}}{v_{1}}=\frac{n+L_{1} / L_{c}}{1+n L_{1} / L_{c}} \frac{1+n L_{2} / L_{c}}{n+L_{2} / L_{c}},
\end{gathered}
$$

where $j=1,2,+,-$, and we have adopted the convention that scaled quantities are denoted with a bar. Furthermore, the finesse $\mathcal{F}_{j}$ has been introduced as the cavity free spectral range $\Delta \nu_{j}^{(F S R)}=1 / \tau_{j}$, divided by the full-width-halfmaximum linewidth $\Delta \nu_{j} \simeq \gamma_{j} / \pi, j=1,2$. The latter approximation is valid in the case of small cavity round-trip losses, which leads to the expressions

$$
\mathcal{F}_{1} \simeq 2 \pi /\left(T+\mathcal{L}_{1}\right)
$$




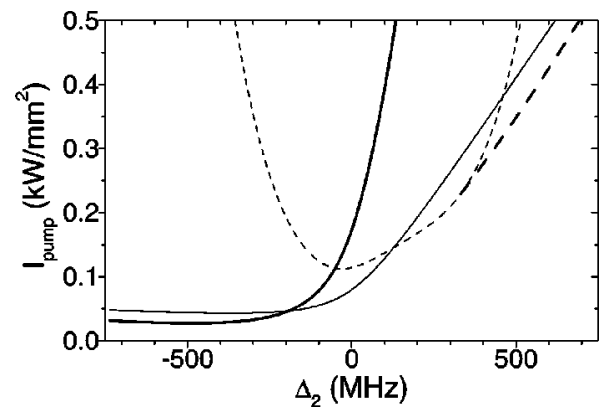

FIG. 10. The threshold pump intensity for the different instabilities in the system. The parameters are specified in the text. Thin curve: parametric instability; bold curve: stationary transverse instability; thin-dashed curve: self-pulsing instability; bold-dashed curve: oscillatory transverse instability.

$$
\mathcal{F}_{2} \simeq 2 \pi / \mathcal{L}_{2}
$$

The intensity of the fundamental pump field is given by

$$
I_{\text {pump }}=\frac{\epsilon_{0} c}{2}\left(\mathcal{E}_{\text {pump }}^{(0)}\right)^{2}=\frac{\epsilon_{0} c n^{3} \pi^{2} \lambda^{2}}{32 d_{\mathrm{eff}}^{2} L_{c}^{2}} \frac{1}{T \mathcal{F}_{1}^{4}} E^{2},
$$

where $\epsilon_{0}$ is the vacuum permittivity and $\lambda$ is the fundamental wavelength.

We consider an experiment consisting of a $1-\mathrm{cm}$ long $\mathrm{LiNbO}_{3}$ crystal placed in a cavity with $L_{1}=L_{2}=1 \mathrm{~cm}$. $\mathrm{LiNbO}_{3}$ can be noncritically phase matched for frequency doubling at the Nd:YAG wavelength $\lambda=1064 \mathrm{~nm}$, where the effective nonlinear coefficient is $d_{\text {eff }}=4.7 \mathrm{pm} / \mathrm{V}$ and the refractive index is $n=2.2$ [34]. Furthermore, the finesse of the fundamental and second-harmonic cavities are assumed to be $\mathcal{F}_{1}=\mathcal{F}_{2}=100$ and the fundamental input coupler transmission $T=3 \%$. From these numbers the cavity linewidth for the fundamental and second harmonic are calculated to be $\Delta \nu_{1}=\Delta \nu_{2}=47 \mathrm{MHz}$. In Fig. 10 the pump intensity necessary to reach threshold for the different instabilities in the system is plotted as a function of the detuning of the second harmonic while the fundamental detuning is fixed at $\Delta_{1}=$ $-368 \mathrm{MHz}$, which corresponds to a scaled detuning $\bar{\Delta}_{1}=$ -2.5. A typical threshold value is about $0.1 \mathrm{~kW} / \mathrm{mm}^{2}$, which should be easily accessible with a pulsed Nd:YAG laser. For comparison, experiments on spatial soliton formation in $\chi^{(2)}$ propagation geometries require on the order of $1 \mathrm{GW} / \mathrm{mm}^{2}[35]$.

The spatial scale of the transverse structure $k_{c}$ and the self-pulsing frequency $\omega_{c}$ can be transformed into physical units as

$$
\begin{aligned}
& k_{c}=\sqrt{\frac{k_{1} \gamma_{1}}{v_{1}}} \bar{k}_{c}=\sqrt{\frac{2 n \pi^{2}}{\lambda \mathcal{F}_{1}} \frac{1}{L_{c}+n L_{1}}} \bar{k}_{c}, \\
& \omega_{c}=\gamma_{1} \bar{\omega}_{c}=\frac{\pi c}{2 \mathcal{F}_{1}} \frac{1}{L_{1}+n L_{c}} \bar{\omega}_{c} .
\end{aligned}
$$

Defining $l_{c}=2 \pi / k_{c}$ as the scale of the spatial modulation, it is favorable to have $l_{c}$ small since this allows more narrow

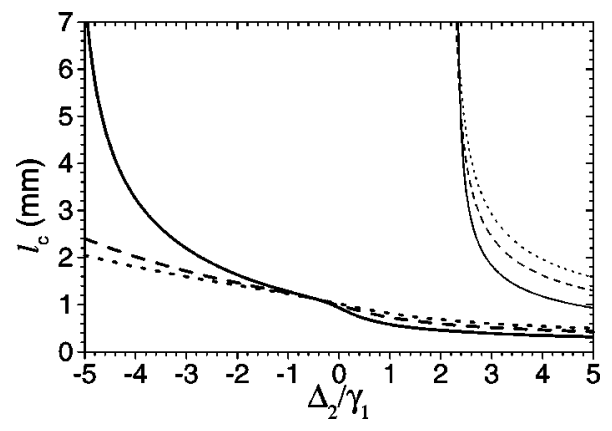

FIG. 11. Spatial scale of the transverse instability displayed as function of the scaled second harmonic detuning. The bold and thin curves are the stationary and oscillatory instabilities, respectively. The full, dashed and dotted curves correspond to a fundamental finesse of 50,100, and 150 , respectively. $\mathcal{F}_{2}=100$.

focusing in the crystal and thus higher intensities. In order to observe pattern formation in an experiment a minimum requirement is that the width of the Gaussian beam is larger than the pattern scale, i.e., $2 w_{0} \geqslant l_{c}$, where $w_{0}$ is the beam radius at the waist. We observe from Eq. (24a) that to obtain a small spatial scale requires a short cavity. It is especially important to minimize the propagation distance outside the crystal since the beam diffracts less in a medium with a reduction factor given by the refractive index. The spatial scales for the stationary and oscillatory transverse structures are plotted in Fig. 11 as a function of the scaled secondharmonic detuning $\bar{\Delta}_{2}$ for different values of the fundamental finesse. The scaled detuning is used in order to relate the detunings of cavities with different values of the fundamental finesse. All other parameters are as described above and in particular $\bar{\Delta}_{1}=-2.5$ for all curves. The typical spatial scale is seen to be about $1 \mathrm{~mm}$.

In Fig. 12 the self-pulsing frequency $\omega_{c}$ is plotted as a function of the second-harmonic detuning for the same value of the fundamental detuning as in Fig. 10. The typical selfpulsing frequency is found to range from about $300 \mathrm{MHz}$ to $1 \mathrm{GHz}$, and is not changed considerably when varying the fundamental detuning.

Finally, the extra degrees of freedom contained in the ratios of the round-trip times $\tau$ and the propagation velocities $v$ should be considered. From an experimental point of view

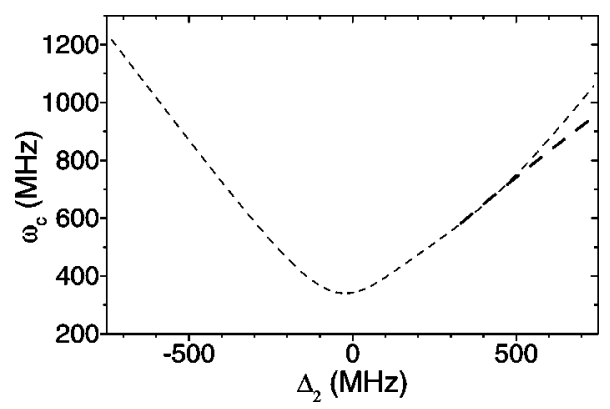

FIG. 12. Self-pulsing frequency $\omega_{c}$ plotted as function of the second-harmonic detuning for $\Delta_{1}=-368 \mathrm{MHz}$. The thin and bold curves correspond to the spatially homogenous and modulated instabilities, respectively. 


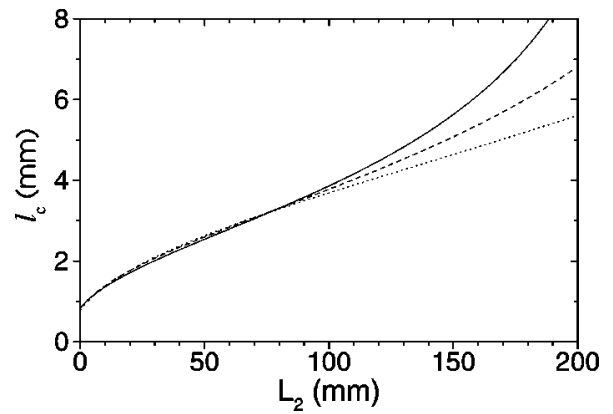

FIG. 13. The spatial scale of the transverse instability as a function of the length of the second harmonic arm $L_{2}$. The lengths of the fundamental arm are $L_{1}=10 \mathrm{~mm}$ (solid), $L_{1}=20 \mathrm{~mm}$ (dashed), and $L_{1}=50 \mathrm{~mm}$ (dotted). The parameters are as in Fig. 10 and with $\bar{\Delta}_{2}=-1.5$.

they may appear to be important since they can be used as convenient tuning parameters. Varying $\tau$ by changing the propagation distances of the fundamental or the second harmonic is seen to be a way to change the loss-rate ratio $\gamma$, which is more easily accessible experimentally than by changing the respective propagation losses of the two fields. The parameter $v$ is found to influence only the diffraction terms and can therefore be used to tune the spatial period of the modulated structure. This may be convenient in order to operate in a regime where the spatial period is small such that a more narrow Gaussian pump beam can be used to enhance the nonlinearity. One way of changing $v$ is to vary the length of the second-harmonic arm $L_{2}$, while keeping the length of the fundamental arm $L_{1}$ fixed. Figure 13 shows the variation of the spatial scale with $L_{2}$. It is observed that substantial tuning of the spatial scale is possible and in general a small value of $L_{2}$ is desirable.

\section{CONCLUSIONS}

This paper concerned pattern formation in doubly resonant SHG in a two-armed cavity configuration of relevance for experimental realizations. As pointed out in previous work the presence of a competing parametric process may influence the pattern formation of the system decisively. A set of coupled cavity mean-field equations generalized to include also unequal phase mismatch for the two competing processes as well as different propagation distances for the fundamental and second harmonic, was presented to model this system. The instabilities were divided into SHG and parametric instabilities, according to the relative position of the SHG instability thresholds to the parametric instability threshold. The parametric instability could be studied in detail by deriving exact analytical solutions that were found to imply complete quenching of spatially modulated intensity structures for positive fundamental detuning. For negative fundamental detuning, however, the parametric solutions could destabilize leading to new phenomena. Here we have considered parametric bistability and self-pulsing that only were found for unequal phase mismatch of the two competing processes. These instabilities were found to lead to formation of a honeycomb pattern and gray solitons. Finally, numerical estimates of the threshold intensity and spatiotemporal scales for the instabilities were given for realistic experimental parameters.

\section{APPENDIX: DETUNING OF THE PARAMETRIC FIELDS}

In this appendix we discuss the relationship between the detuning of the fundamental and parametric fields. Energy conservation requires

$$
2 \omega_{1}=\omega_{+}+\omega_{-},
$$

where $\omega_{j}$ is the angular frequency of field $j$. In a monolithic resonator containing a dispersive crystal there are a set of resonances at vacuum wavelengths $\lambda_{p}$ satisfying

$$
\frac{2 L_{c} n_{p}}{\lambda_{p}}=p
$$

where $L_{c}$ is the crystal length, $p$ is an integer, and $n_{p}$ $=n\left(\lambda_{p}\right)$ is the crystal's index of refraction at $\lambda_{p}$. For simplicity we have neglected the distance $L_{1}$ in Fig. 1 . The corresponding set of resonant frequencies is

$$
\omega_{p}=2 \pi \frac{c}{\lambda_{p}}=p \frac{\pi c}{L_{c} n_{p}} .
$$

Define detunings as $\delta_{j}=\omega_{j}-\omega_{p_{j}}, j=1,+,-$. Then Eq. (A1) implies

$$
2 \omega_{p_{1}}-\omega_{p_{+}}-\omega_{p_{-}}=\delta_{+}+\delta_{-}-2 \delta_{1} .
$$

Assuming that the parametric fields are emitted symmetrically, we have $\omega_{p_{+}}=\omega_{p_{1} \pm m}$, where $m$ is an integer labeling the axial mode the parametric fields are nearly resonant with. Hence, the left-hand side of (A4) can be written as

$$
\mathrm{lhs}=\frac{2 \pi c}{\lambda_{p_{1}}}\left[2-\frac{n_{p_{1}}}{n_{p_{+}}}-\frac{n_{p_{1}}}{n_{p_{-}}}-\frac{m}{p_{1}}\left(\frac{n_{p_{1}}}{n_{p_{+}}}-\frac{n_{p_{1}}}{n_{p_{-}}}\right)\right] .
$$

Converting to normalized detunings using the same scalings as in Eqs. (1) then gives

$$
\begin{aligned}
\Delta_{+}+\Delta_{-}-2 \Delta_{1}= & \frac{8 \pi n_{p_{1}} L_{c}}{\lambda_{p_{1}}} \frac{1}{T+\mathcal{L}_{1}} \\
& \times\left[2-\frac{n_{p_{1}}}{n_{p_{+}}}-\frac{n_{p_{1}}}{n_{p_{-}}}-\frac{m}{p_{1}}\left(\frac{n_{p_{1}}}{n_{p_{+}}}-\frac{n_{p_{1}}}{n_{p_{-}}}\right)\right] .
\end{aligned}
$$

When $m=0, n_{p_{+}}=n_{p_{-}}=n_{p_{1}}$ and $\Delta_{+}+\Delta_{-}-2 \Delta_{1}=0$. At finite $m$ this relation is only approximate. As an example, consider $\mathrm{LiNbO}_{3}$ at room temperature, $L_{c}=1 \mathrm{~cm}, T=\mathcal{L}_{1}$ $=0.03, \lambda_{p_{1}}=1.06 \mu \mathrm{m}$, and $m \sim 20$ (corresponding to a wavelength separation between the parametric beams of about $1 \mathrm{~nm}$ ). Equation (A6) then gives $\Delta_{+}+\Delta_{-}-2 \Delta_{1}=$ 
-0.1 , where the refractive indices have been calculated using the Sellmeier equations given in Ref. [34]. In practice the axial mode shift $m$, and the selected detuning of the parametric fields from the nearest cavity resonances, depend on the interaction of the phase mismatch of the fundamental and the cavity tuning in a rather complicated way [10]. Thus even though the fundamental is close to phase matching there may be a large shift of the parametric frequencies. Analysis of that situation, which implies arbitrary parametric detunings, is not included in this paper.
[1] G.-L. Oppo, M. Brambilla, and L.A. Lugiato, Phys. Rev. A 49, 2028 (1994); G.-L. Oppo, M. Brambilla, D. Camesasca, A. Gatti, and L.A. Lugiato, J. Mod. Opt. 41, 1151 (1994).

[2] K. Staliunas, J. Mod. Opt. 42, 1261 (1995).

[3] S. Longhi, Phys. Rev. A 53, 4488 (1996).

[4] C. Etrich, U. Peschel, and F. Lederer, Phys. Rev. Lett. 79, 2454 (1997).

[5] C. Etrich, U. Peschel, and F. Lederer, Phys. Rev. E 56, 4803 (1997).

[6] S. Longhi, Phys. Rev. E 59, R24 (1999); Phys. Rev. A 59, 4021 (1999).

[7] P. Lodahl and M. Saffman, Phys. Rev. A 60, 3251 (1999).

[8] P. Lodahl and M. Saffman, Opt. Commun. 184, 493 (2000).

[9] P. Lodahl, M. Bache, and M. Saffman, Opt. Lett. 25, 654 (2000).

[10] S. Schiller and R.L. Byer, J. Opt. Soc. Am. B 10, 1696 (1993); S. Schiller, G. Breitenbach, R. Paschotta, and J. Mlynek, Appl. Phys. Lett. 68, 3374 (1996).

[11] M.A.M. Marte, Phys. Rev. A 49, R3166 (1994).

[12] M.A.M. Marte, Phys. Rev. Lett. 74, 4815 (1995).

[13] A.G. White, P.K. Lam, M.S. Taubman, M.A.M. Marte, S. Schiller, D.E. McClelland, and H.-A. Bachor, Phys. Rev. A 55, 4511 (1997).

[14] P. Lodahl, M. Bache, and M. Saffman, Phys. Rev. Lett. 85, 4506 (2000).

[15] M. Vaupel, A. Maitre, and C. Fabre, Phys. Rev. Lett. 83, 5278 (1999).

[16] Z.Y. Ou and H.J. Kimble, Opt. Lett. 18, 1053 (1993).

[17] G.J. de Valcárcel, Phys. Rev. A 56, 1542 (1997).

[18] K.J. McNeil, P.D. Drummond, and D.F. Walls, Opt. Commun. 27, 292 (1978); P.D. Drummond, K.J. McNeil, and D.F. Walls, Opt. Acta 27, 321 (1980).
[19] C. Fabre and S. Reynaud, in Fundamental Systems in Quantum Optics, edited by J. Dalibard, J.M. Raimond, and J. Zinn-Justin (Elsevier Science, Amsterdam, 1992).

[20] K. Staliunas and V.J. Sánchez-Morcillo, Opt. Commun. 139, 306 (1997).

[21] S. Longhi, Phys. Scr. 56, 611 (1997).

[22] D.V. Skryabin and W.J. Firth, Opt. Lett. 24, 1056 (1999).

[23] S. Longhi, Opt. Commun. 149, 335 (1998).

[24] V.J. Sánchez-Morcillo, E. Roldán, G.J. de Valcárcel, and K. Staliunas, Phys. Rev. A 56, 3237 (1997).

[25] D.V. Skryabin, A.R. Champneys, and W.J. Firth, Phys. Rev. Lett. 84, 463 (2000).

[26] A. Eschmann and M.A.M. Marte, Quantum Semiclassic. Opt. 9, 247 (1997).

[27] C.M. Savage and D.F. Walls, Opt. Acta 30, 557 (1983).

[28] K. Schneider and S. Schiller, Opt. Lett. 22, 363 (1997).

[29] N.N. Rosanov and G.V. Khodora, J. Opt. Soc. Am. B 7, 1057 (1990).

[30] S. Trillo, M. Haelterman, and A. Sheppard, Opt. Lett. 22, 970 (1997).

[31] G.J. de Valcárcel, E. Roldán, and K. Staliunas, Opt. Commun. 181, 207 (2000).

[32] D. Michaelis, U. Peschel, and F. Lederer, Opt. Lett. 23, 1814 (1998).

[33] M. Hoyuelos, P. Colet, M. San Miguel, and D. Walgraef, Phys. Rev. E 58, 2992 (1998).

[34] V.G. Dmitriev, G.G. Gurzadyan, and D.N. Nikogosyan: Handbook of Nonlinear Optical Crystals (Springer-Verlag, New York, 1997).

[35] W.E. Torruellas, Z. Wang, D.J. Hagan, E.W. VanStryland, G.I. Stegeman, L. Torner, and C.R. Menyuk, Phys. Rev. Lett. 74, 5036 (1995). 